\section{The variable manifestations of disease in pyruvate kinase deficiency and their management}

\author{
Hanny Al-Samkari, ${ }^{1}$ Eduard J. van Beers, ${ }^{2}$ Kevin H.M. Kuo, ${ }^{3}$ Wilma Barcellini, ${ }^{4}$ \\ Paola Bianchi, ${ }^{4}$ Andreas Glenthøj, ${ }^{5}$ María del Mar Mañú Pereira, ${ }^{6}$ Richard van \\ Wijk, ${ }^{7}$ Bertil Glader ${ }^{8}$ and Rachael F. Grace ${ }^{9}$
}

${ }^{1}$ Division of Hematology, Massachusetts General Hospital, Harvard Medical School, Boston, MA, USA; ${ }^{2}$ Van Creveldkliniek, University Medical Centre Utrecht, University of Utrecht, Utrecht, the Netherlands; ${ }^{3}$ Division of Hematology, University of Toronto, University Health Network, Toronto, Ontario, Canada; ${ }^{4}$ UOS Ematologia, Fisiopatologia delle Anemie, Fondazione IRCCS Ca' Granda Ospedale Maggiore Policlinico, Milan, Italy; ${ }^{5}$ Department of Hematology, Herlev and Gentofte Hospital, Herlev, Denmark; ${ }^{\circ}$ Translational Research in Rare Anaemia Disorders, Vall d'Hebron Institut de Recerca (VHIR), Barcelona, Spain; ${ }^{7}$ Department of Clinical Chemistry \& Hematology, University Medical Center Utrecht, Utrecht University, Utrecht, the Netherlands; ' ${ }^{2}$ Lucile Packard Children's Hospital, Stanford University School of Medicine, Palo Alto, CA, USA and 'Dana/Farber Boston Children's Cancer and Blood Disorders Center, Harvard Medical School, Boston, MA, USA.
$\mathrm{P}$ yruvate kinase deficiency ( $\mathrm{PKD}$ ) is the most common cause of chronic hereditary non-spherocytic hemolytic anemia and results in a broad spectrum of disease. The diagnosis of PKD requires a high index of suspicion and judicious use of laboratory tests that may not always be informative, including pyruvate kinase enzyme assay and genetic analysis of the PKLR gene. A significant minority of patients with PKD have occult mutations in non-coding regions of PKLR which are missed on standard genetic tests. The biochemical consequences of PKD result in hemolytic anemia due to red cell pyruvate and ATP deficiency while simultaneously causing increased red cell 2,3-diphosphoglycerate, which facilitates oxygen unloading. This phenomenon, in addition to numerous other factors such as genetic background and differences in splenic function result in a poor correlation between symptoms and degree of anemia from patient to patient. Red cell transfusions should, therefore, be symptom-directed and not based on a hemoglobin threshold. Patients may experience specific complications, such as paravertebral extramedullary hematopoiesis and chronic debilitating icterus, which require personalized treatment. The decision to perform splenectomy or hematopoietic stem cell transplantation is nuanced and depends on disease burden and long-term outlook given that targeted therapeutics are in development. In recognition of the complicated nature of the disease and its management and the limitations of the PKD literature, an international working group of ten PKD experts convened to better define the disease burden and manifestations. This article summarizes the conclusions of this working group and is a guide for clinicians and investigators caring for patients with PKD.

\section{Introduction}

Pyruvate kinase deficiency (PKD) is the most common cause of chronic hereditary non-spherocytic hemolytic anemia, with a prevalence reported to be between 1:20,000 and 1:300,000 in Caucasian populations and a higher prevalence in areas in which malaria is endemic. ${ }^{1-4}$ Despite its well-described global geographical distribution, its incidence in large areas of the world remains unknown.

Following the identification by Selwyn and Dacie in 1954 of a patient with hereditary hemolytic anemia whose erythrocytes were not rescued by glucose but were rescued by adenosine triphosphate (ATP) in an ex vivo hemolysis assay, it was
Ferrata Storti Foundation
Haematologica 2020
Volume 105(9):2229-2239

\section{Correspondence:}

HANNY AL-SAMKARI

hal-samkari@mgh.harvard.edu

Received: October 21, 2019.

Accepted: January 20, 2020.

Pre-published: March 12, 2020.

doi:10.3324/haematol.2019.240846

(C)2020 Ferrata Storti Foundation

Material published in Haematologica is covered by copyright. All rights are reserved to the Ferrata Storti Foundation. Use of published material is allowed under the following terms and conditions:

https://creativecommons.org/licenses/by-nc/4.0/legalcode. Copies of published material are allowed for personal or internal use. Sharing published material for non-commercial purposes is subject to the following conditions:

https://creativecommons.org/licenses/by-nc/4.0/legalcode, sect. 3. Reproducing and sharing published material for commercial purposes is not allowed without permission in writing from the publisher. 
recognized that glycolytic defects could lead to hemolytic anemia. ${ }^{5}$ PKD was first described as a cause of hereditary hemolytic anemia in 1961 by Valentine and colleagues. ${ }^{6}$ Pyruvate kinase (PK) catalyzes the conversion of phosphenolpyruvate to pyruvate and is the rate-limiting enzyme in erythrocyte energy production, which is an exclusively anaerobic process. As only one of two glycolytic enzymes that generates ATP, homozygous or compound heterozygous loss-of-function mutations in the PKLR gene, which encodes erythrocyte PK, result in erythrocyte ATP shortage. This ATP deficiency presumably results in a reduced capacity to maintain the red cell membrane and diminished erythrocyte deformability, resulting in a shortened lifespan and destruction in the spleen. Because patients experience mainly extravascular hemolysis, splenectomy frequently improves anemia. A striking difference between PKD and most other hereditary hemolytic anemias is the dramatic post-splenectomy reticulocytosis characteristic of $\mathrm{PKD}$, which typically increases $50 \%$ or more over pre-splenectomy counts. Reticulocytes have a much higher ATP requirement as compared with mature red cells but can rely on oxidative phosphorylation for energy. However, in the hypoxic splenic environment, PKdeficient reticulocytes must rely on glycolysis, which does not meet the ATP needs, leading to hemolysis. ${ }^{8-10}$ In the absence of the spleen, reticulocyte survival increases.

The clinical manifestations of PKD are heterogenous and the severity varies considerably, from fetal hydrops with intrauterine demise to incidentally discovered asymptomatic fully compensated hemolysis to a severe transfusion-dependent anemia from birth through old age. ${ }^{11-13}$ Patients' symptoms often do not correlate with the severity of anemia, adding an additional dimension of complexity in disease management. ${ }^{14}$ Complications include iron overload, pulmonary hypertension, endocrinopathies, osteoporosis and bone fractures, extramedullary hematopoiesis, gallstones, and lower extremity ulcers, among others. ${ }^{11} \mathrm{PKD}$ is characterized by a high prevalence of iron overload regardless of whether patients require regular transfusions, necessitating monitoring in all patients and frequent institution of iron chelation therapy. ${ }^{15}$

Beyond splenectomy and hematopoietic stem cell transplantation, treatment has been largely supportive from the time PKD was first described. ${ }^{16,17}$ This is rapidly changing. A small molecule allosteric activator of PK is under development, with safety and efficacy demonstrated in phase I and II trials, ${ }^{18,19}$ it is currently being investigated in phase III trials. Following on the success of gene therapy in animal PKD models ${ }^{20}$ and humans with thalassemia and other hematologic disorders, ${ }^{21,22}$ a clinical trial of gene therapy for PKD was launched in 2019. Now more than ever before, it is critical for hematologists to elucidate the specific diagnoses of patients with congenital hemolytic anemias, including PKD, and institute proper interventions. The promise of effective targeted therapies has greatly revived interest in $\mathrm{PKD}$, but published data remain limited and no evidence-based guidelines for the management of these patients exist. This article, developed and written by ten international experts in $\mathrm{PKD}$, reviews the manifestations and spectrum of disease in patients and highlights the most common, most important, and most challenging presentations of this disease. To develop this report, this PKD Burden of Disease working group had multiple focused online discussions over the course of 1 year and an in-person meeting in San Diego (CA, USA). Given that many providers have limited experience caring for patients with PKD, hypothetical case presentations based on the collective PKD patient care experience of the working group are included to introduce each section and illustrate the key signs, symptoms, and complications which outline the scope and spectrum of disease in PKD.

\section{Diagnostic challenges}

\section{The newly-diagnosed adult patient}

CASE: A 29-year old man with a history of ulcerative colitis, inflammatory anemia, and cholecystitis (cholecystectomy performed at the age of 27) is referred for further workup of hemolytic anemia with a negative direct antiglobulin test. He underwent colectomy at the age of 17 for ulcerative colitis. Following colectomy, his chronic anemia (hemoglobin 9.0-11.0 $\mathrm{g} / \mathrm{dL}$ ) improved to a new baseline of $11.5-12.5 \mathrm{~g} / \mathrm{dL}$. He has long-standing hyperbilirubinemia that has been attributed to Gilbert syndrome, although genetic confirmation of this has not been performed. Currently his laboratory evaluation is remarkable for the following: hemoglobin $10.9 \mathrm{~g} / \mathrm{dL}$, mean cell volume $106 \mathrm{fL}$, absolute reticulocyte count 1,030 $\times 10^{9} / \mathrm{L}$, indirect bilirubin $3.7 \mathrm{mg} / \mathrm{dL}$, lactate dehydrogenase $476 \mathrm{U} / \mathrm{L}$, and undetectable haptoglobin. The direct antiglobulin test is negative. A peripheral blood film is nonspecific and hemoglobin electrophoresis is unremarkable. Testing for paroxysmal nocturnal hemoglobinuria, erythrocyte membrane defects, and glucose-6-phosphate dehydrogenase deficiency is negative. PK activity is $1.2 \mathrm{U} / g$ hemoglobin (reference range, 6.7-14.3 U/g hemoglobin). A diagnosis of PKD is confirmed by genetic testing revealing compound heterozygosity for two missense mutations in the PKLR gene: c.1091G>A (p.Gly364Asp) and c.1529G>A (p.Arg510Gln).

Patients with PKD who are regularly transfused or have severe hemolytic anemia are typically diagnosed in the neonatal period or in early childhood. Patients who have never or rarely been transfused, however, are frequently diagnosed as adults. ${ }^{23}$ These patients typically have mildto-moderate anemia that can be misdiagnosed as thalassemia trait (without genetic confirmation), iron deficiency (often in a menstruating woman), more common hemolytic anemias (e.g. hereditary spherocytosis), or inflammatory anemia. Patients can also evade early diagnosis because of normal or near normal hemoglobin concentrations in the setting of well-compensated hemolysis. Complications resulting from $\mathrm{PKD}$, such as hyperbilirubinemia, gallstones, and iron overload, can often be mistaken as discrete diagnostic entities attributed to other causes, such as Gilbert syndrome or hereditary hemochromatosis.

Recognition of even the mildest forms of disease is important for several reasons. Although the frequency of certain complications is highest in patients with two drastic PKLR mutations, many complications, including hemolysis, gallstones, iron overload, and aplastic crises are not uncommon in patients with two missense mutations and mild anemia. ${ }^{11}$ Folic acid supplementation is recommended in essentially all patients to prevent deficiency due to rapid cell turnover; recognition of increased folate requirements is particularly important in women of childbearing age. Iron overload is common even in patients who never receive red cell transfusion due to chronic hemolysis ${ }^{15}$ and may lead to 


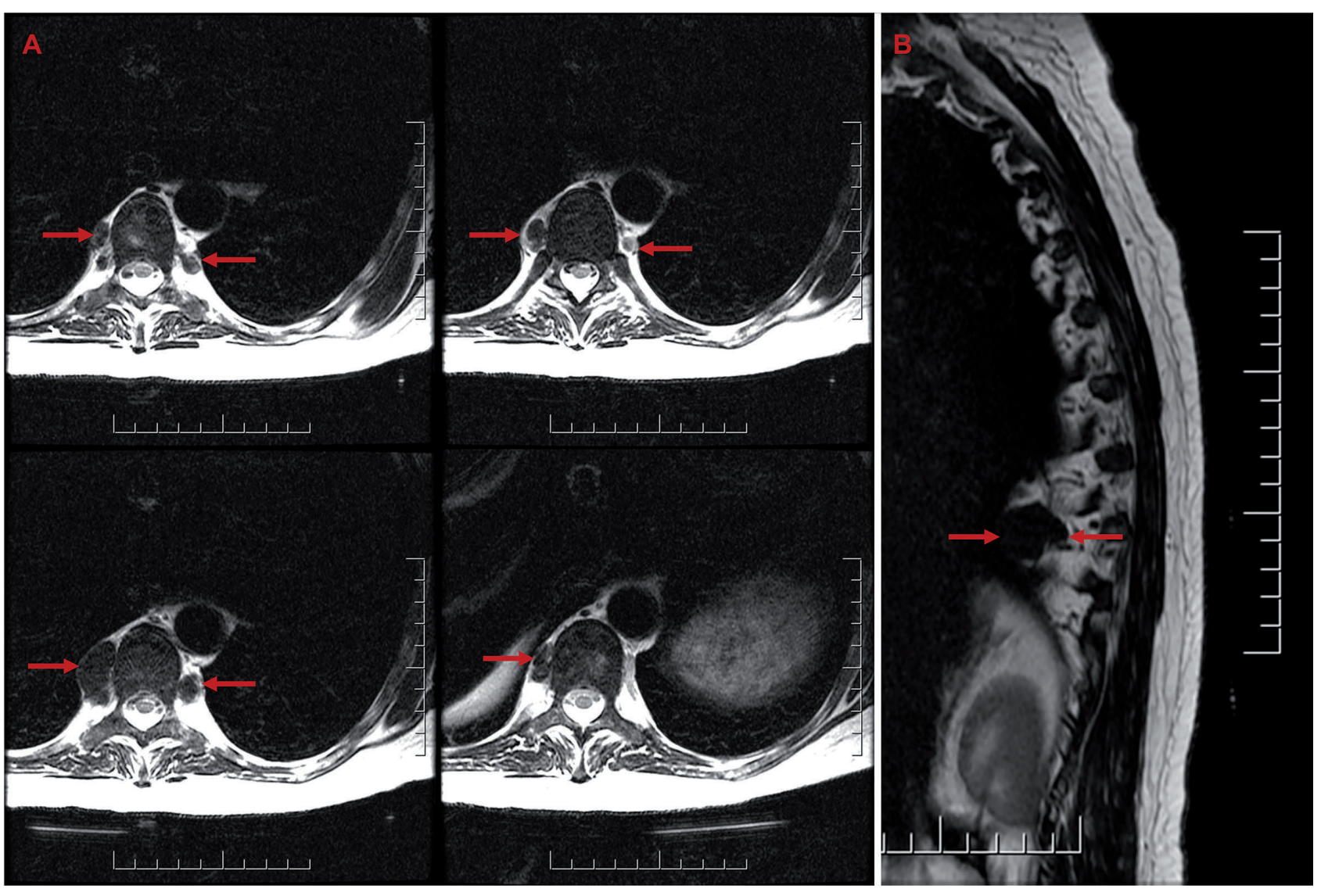

Figure 1. Paravertebral extramedullary hematopoietic masses in pyruvate kinase deficiency. (A) Multiple transverse sections demonstrating paravertebral masses (arrows) in close proximity to nerve roots. (B) Sagittal section demonstrating a large paravertebral mass (bounded by arrows) extending from the vertebra.

liver dysfunction, cardiac dysfunction, or endocrinopathies if not addressed. A small but significant proportion of patients can develop extramedullary hematopoiesis. ${ }^{11}$ Extramedullary hematopoietic masses in PKD are often paravertebral, ${ }^{24-26}$ as illustrated in Figure 1. These masses can enlarge over time and can result in nerve root compression resulting in neurological compromise, including paralysis, if left untreated..$^{27,28}$ Although evidence is lacking, some members of the working group treat patients with progressing paravertebral masses with chronic red cell transfusion to suppress the growth of the masses. Extramedullary hematopoietic masses can also be mistaken for malignant tumors, especially in undiagnosed patients. As in other hemolytic anemias, patients with PKD can develop lowerextremity ulcers, usually medial in association with the medial malleolus, ${ }^{29}$ which can be slow to heal or even fail to heal (Figure 2). There are no data to guide the management of leg ulcers in $\mathrm{PKD}$, so ulcers are managed similarly to those seen in sickle cell disease or thalassemia. ${ }^{30,31}$

Once PKD has been diagnosed, screening and regular monitoring for complications from chronic hemolysis should be initiated, since many complications, such as iron overload, can be asymptomatic. ${ }^{15}$ The type and frequency of screening vary between institutions, but the screening is usually directed at complications that carry high morbidity if untreated. These include iron overload, extramedullary hematopoiesis, osteopenia, osteoporosis, gallstones, and pulmonary hypertension. Table 1 shows our consensus approach to screening in patients with $\mathrm{PKD}$.

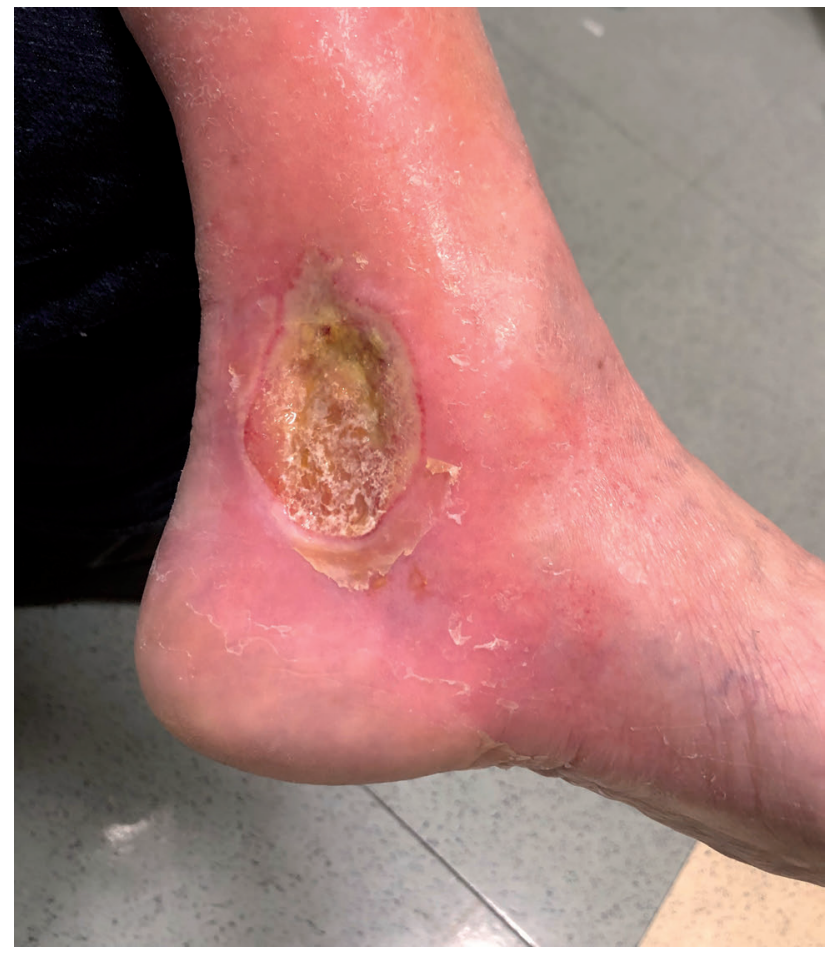

Figure 2. Lower extremity non-healing ulcer in an adult with pyruvate kinase deficiency. Note the location posterior to the medial malleolus. 


\section{Complexity of enzymatic and genetic diagnosis} of pyruvate kinase deficiency

CASE: A 24-month old boy previously diagnosed with hereditary xerocytosis and glucose-6-phosphate dehydrogenase deficiency is seen for a second opinion. He has had severe transfusion-dependent hemolytic anemia since birth. On evaluation, $P K$ enzyme activity is in the normal range, but with a low PK/hexokinase ratio. There is no family history of anemia. On evaluation of the patient's parents, the patient's mother has PK enzyme activity approximately $50 \%$ of normal and a low PK/hexokinase ratio and the patent's father has normal PK enzyme activity and PK/hexokinase ratio.

Genetic testing is performed and the patient is found to be a compound heterozygote for c.1091G>A (p.Gly364Asp) and c.1529G>A (p.Arg510Gln) mutations in exons 8 and 11 of $P K L R$, respectively. As these are known pathogenic mutations, the diagnosis of PKD is confirmed. PKLR analysis of the patient's mother showed the c.1091G>A mutation. Surprisingly,
PKLR analysis of the patient's father revealed two wildtype alleles and no evidence of a PKLR c.1529G>A mutation (paternity was confirmed via additional testing).

Further testing was performed to identify a possible somatic mutation in PKLR in the father. Massive parallel sequencing of the region encompassing nucleotide c.1529 of PKLR was performed on paternal genomic DNA isolated from peripheral blood, a buccal swab, urine, and semen. Sequencing of the same region was performed on genomic DNA isolated from peripheral blood from the patient, his mother and paternal grandparents. The results of this testing are shown in Table 2.

The presence of the $c .1529 \mathrm{G}>A$ mutation was demonstrated in $D N A$ of all tissues tested from the father. DNA from the paternal grandparents showed absence of the c.1529G>A mutation, thereby confirming the post-zygotic origin of the $c .1529 \mathrm{G}>A$ mutation in the father. Altogether, these results confirmed that the father is a mosaic for the $c .1529 \mathrm{G}>A$ mutation in PKLR.

PKD should be suspected in patients with an unex-

Table 1. Our consensus approach to routine screening of the patient with pyruvate kinase deficiency.

$\begin{array}{ll}\text { Condition } & \text { Recommended Screening } \\ \text { Cholelithiasis } & \begin{array}{l}\text { Consider screening for gallstones with interval ultrasound examinations; discuss cholecystectomy with patient if gallstones } \\ \text { are observed }\end{array}\end{array}$

Cholecystectomy should be considered at the time of splenectomy in patients undergoing splenectomy even in the absence of gallstones due to the high rate of gallstones in patients with pyruvate kinase deficiency (both in general and following splenectomy) ${ }^{40}$ patients should be counseled on the risk of intrahepatic cholestasis

Viral infections Human immunodeficiency virus and hepatitis $\mathrm{C}$ annually is reasonable if receiving blood transfusions in certain countries (risk varies depending on country)

Baseline parvovirus B19 serology if parvovirus serological status unknown

Iron overload $\quad$ Non-transfused or minimally transfused patients: ferritin yearly; R2 or T2* magnetic resonance imaging of liver and heart once in early adulthood or when ferritin $>500 \mu \mathrm{g} / \mathrm{L}$ with follow-up interval dependent on findings

Regularly transfused patients: ferritin every 6 months (every 3 months if receiving chelation therapy); R2 or T2* magnetic resonance imaging yearly

Osteopenia $\quad 25$-hydroxy-vitamin D levels yearly; if low, replete and re-check after 8 weeks of vitamin D repletion DEXA baseline in early adulthood, with follow-up interval dependent on findings

Endocrinopathies Thyroid-stimulating hormone, sex hormones and fructosamine ${ }^{a}$ yearly; can forego screening or increase screening interval in patients with no evidence of iron overload

Pulmonary hypertension Echocardiogram once after the age of 30 or prior to pregnancy; otherwise perform only for concerning symptoms

Extramedullary Perform imaging only for concerning symptoms; have a high index of suspicion for paravertebral extramedullary hematopoiesis hematopoiesis $\quad$ in cases of neuropathy or unexplained pain

Fructosamine should be used instead of hemoglobin Alc for diabetes mellitus screening in patients with hemolytic anemias.

Table 2. Results of massive parallel sequencing of the region encompassing nucleotide c.1529 of PKLR on genomic DNA isolated from peripheral blood, buccal swab, urine, and semen from members of the family of the patient in the case described in "Complexity of enzymatic and genomic diagnosis in pyruvate kinase deficiency"

\begin{tabular}{|c|c|c|c|c|}
\hline Individual & Sample & $\begin{array}{l}\text { PKLR genotype by Sanger sequencing } \\
\text { for nucleotide c.1529 }\end{array}$ & $\begin{array}{l}\text { Massive par } \\
\text { PKLR genotype } \\
\text { for nucleotide c.1529 }\end{array}$ & $\begin{array}{l}\text { Muencing } \\
\text { Mutated allele } \\
\text { frequency (\%) }\end{array}$ \\
\hline Patient & Peripheral blood & c. $1529 G>A / w t$ & c. $1529 G>A / w t$ & 48.6 \\
\hline Mother & Peripheral blood & wt/wt & wt/wt & 0 \\
\hline Father & $\begin{array}{l}\text { Peripheral blood } \\
\text { Urine } \\
\text { Buccal swab } \\
\text { Semen }\end{array}$ & $\begin{array}{c}\mathrm{wt} / \mathrm{wt} \\
\mathrm{wt} / \mathrm{wt} \\
\mathrm{wt} / \mathrm{wt} \\
\mathrm{c} .1529 \mathrm{G}>\mathrm{A} / \mathrm{wt}\end{array}$ & $\begin{array}{l}c .1529 G>A / w t \\
c .1529 G>A / w t \\
c .1529 G>A / w t \\
c .1529 G>A / w t\end{array}$ & $\begin{array}{c}5.1 \\
10.3 \\
16.73 \\
21.76\end{array}$ \\
\hline Paternal grandfather & Peripheral blood & wt/wt & wt/wt & 0 \\
\hline Paternal grandmother & Peripheral blood & $\mathrm{wt} / \mathrm{wt}$ & wt/wt & 0 \\
\hline
\end{tabular}

wt: wildtype. 
plained chronic Coombs-negative hemolytic anemia following an unrevealing standard hemolytic anemia evaluation..$^{23}$ Tests to be considered in this situation are outlined in Table 3, and our stepwise approach to the diagnosis of a patient with chronic unexplained hemolytic anemia is detailed in Figure 3. The first test to perform in cases of suspected PKD is the PK enzyme assay. While it is a useful and inexpensive screening test, PK enzyme activity assays have a number of limitations. Recent transfusion can result in falsely normal enzyme activity. ${ }^{17,23} \mathrm{PK}$ enzyme activity is red cell age-dependent, and so cases of suspected PKD with a normal PK enzyme assay should also be evaluated further via calculation of the PK/hexokinase ratio. ${ }^{32}$ This ratio relates $\mathrm{PK}$ activity to the activity of hexokinase, another red cell age-dependent enzyme used as an internal standard. A decreased $\mathrm{PK} /$ hexokinase ratio is suspicious for PKD and should be investigated further with genetic testing. ${ }^{23}$ Of clinical relevance, PK enzyme activity has not been shown to correlate with disease severity.,

In addition, this case demonstrates the complexity of genetic diagnosis in $\mathrm{PKD}$. Over 300 functional mutations in PKLR have been described. ${ }^{33,34}$ While most cases can be diagnosed with standard PKLR exon sequencing of the patient, occasionally more comprehensive analysis are necessary to confirm the diagnosis or allow for family planning in subsequent pregnancies. This includes screening for parental somatic mutations in cases in which an apparent de novo mutation is identified..$^{35}$ In more routine cases, in which a couple is planning to conceive and one partner is a confirmed carrier of PKD (as occurs in all children of affected individuals), testing of the partner with an unconfirmed PKLR mutation status should be through sequencing rather than testing $\mathrm{PK}$ enzyme activity, as many carriers will have normal PK activity. An analysis of PK enzyme activity in 31 family studies (mother, father, and patient) performed by three of the authors (RvW, PB,
MMP, unpublished observation) revealed eight cases in which a carrier had normal PK activity. Therefore, in this setting enzymatic testing alone to identify possible carriers is inadequate and genetic testing is advised.

\section{Occult mutations in pyruvate kinase deficiency}

CASE: A 47-year old woman with PKD on regular transfusions following splenectomy and cholecystectomy is referred for confirmation of her diagnosis after genetic testing revealed only heterozygosity for the c.460G>A (p.Ala154Thr) mutation. She was initially diagnosed with PKD over 30 years ago and despite splenectomy in childhood has required regular transfusions $(2$ units every 3 weeks). PK enzyme activity measured immediately prior to transfusion is $2.5 \mathrm{U} / g$ hemoglobin (reference range, 6.7$14.3 \mathrm{U} / \mathrm{g}$ hemoglobin). The c.460G $>$ A mutation was inherited from the patient's mother who had normal $P K$ activity $18.1 \mathrm{U} / g$ hemoglobin) and a normal $P K /$ hexokinase ratio of 9.0. No other relatives are available for analysis. To exclude the possibility of other causes of hereditary hemolytic anemia, a targeted next-generation sequencing gene panel analysis was performed, which did not identify additional mutations in 46 genes associated with hereditary hemolytic anemias. Given the severity of her disease, she was interested in considering a clinical trial of PKD-directed treatment but was excluded because she had only one mutated PKLR allele.

While it is important to confirm an enzymatic diagnosis of PKD with genetic testing, ${ }^{23}$ up to $10 \%$ of patients with a diagnosis of PKD will have only one mutation identified on standard PKLR exome sequencing. ${ }^{36}$ It is recognized that $P K L R$ intron splicing-associated mutations are sometimes found in patients with single or no PKLR coding mutations. ${ }^{37}$ In one study of 13 kindreds with hereditary non-spherocytic hemolytic anemia and either single or no identified mutations in PKLR coding regions, whole genome sequencing identified five kindreds with unique PKLR deep intronic mutations predicted to perturb normal

Table 3. Advanced diagnostic workup of a patient with unidentified Coombs-negative hemolytic anemia to be considered after basic testing (peripheral blood film, hemoglobin electrophoresis, etc.). Often many of these tests will be indicated in such a patient and may be obtained in a stepwise fashion to diagnose the underlying disorder. Our consensus stepwise approach is given in Figure 1.

Test

Pyruvate kinase enzyme assay, other glycolytic enzyme testing, pyrimidine 5'-nucleotidase testing

Extended direct antiglobulin testing (e.g. IgA detection, elution)

Osmotic gradient ektacytometry, osmotic fragility test, eosin-5-maleimide binding, genetic testing for red cell membrane mutations

Flow cytometry for CD55 and CD59, multiparameter fluorescent aerolysin-based flow cytometry Ceruloplasmin

ADAMTS13 activity

Genetic testing for unstable hemoglobin variants

Targeted next-generation sequencing panels

\section{Situations to Consider}

Chronic/lifelong hemolytic anemia with bland peripheral blood film, possible splenomegaly; certain glycolytic defects may present with other pathological features (e.g. severe neuromuscular symptoms in triosephosphate isomerase deficiency, myopathy in phosphofructokinase deficiency)

Basophilic stippling of red cells common in pyrimidine 5'-nucleotidase deficiency

Evidence of autoimmune hemolysis (e.g. spherocytes, microspherocytes) but standard direct antiglobulin test negative for IgG and C3

Possible red cell membrane disorders (e.g. hereditary spherocytosis, hereditary elliptocytosis, hereditary xerocytosis) or congenital dyserythropoietic anemias

Hemolysis with thrombosis and/or other cytopenias

Screening for Wilson disease in a patient with unidentified Coombs-negative hemolytic anemia and psychiatric/neurological symptoms, evidence of liver dysfunction, and/or Kayser-Fleischer rings Concomitant thrombocytopenia in a patient with chronic unidentified hemolysis is concerning for congenital thrombotic thrombocytopenic purpura (Upshaw-Schulman syndrome)

Chronic/lifelong hemolytic anemia with bland peripheral blood film or basophilic stippling of red cells, possible splenomegaly

Cases with unexplained hemolytic anemia after comprehensive hematologic testing or when hematologic testing cannot be performed accurately (i.e. in recently-transfused patients, neonates, or shipped samples) 
mRNA processing. ${ }^{38}$ Additional mutations in other regions involved in PKLR gene expression may also play a role in the pathogenesis of $\mathrm{PKD} .{ }^{39}$ In addition to lack of diagnosis, patients with only one identifiable mutation in PKLR may be excluded from clinical trials, which use the presence of two mutated PKLR alleles for eligibility. This is what occurred in the case described above: the patient described clearly has a clinical and enzymatic diagnosis of $\mathrm{PKD}$, but standard exome sequencing-based genetic testing has failed to elucidate her genotype. Such patients with high suspicion of PKD as the cause of chronic hemolysis (e.g. persistently low PK enzyme activity) should be

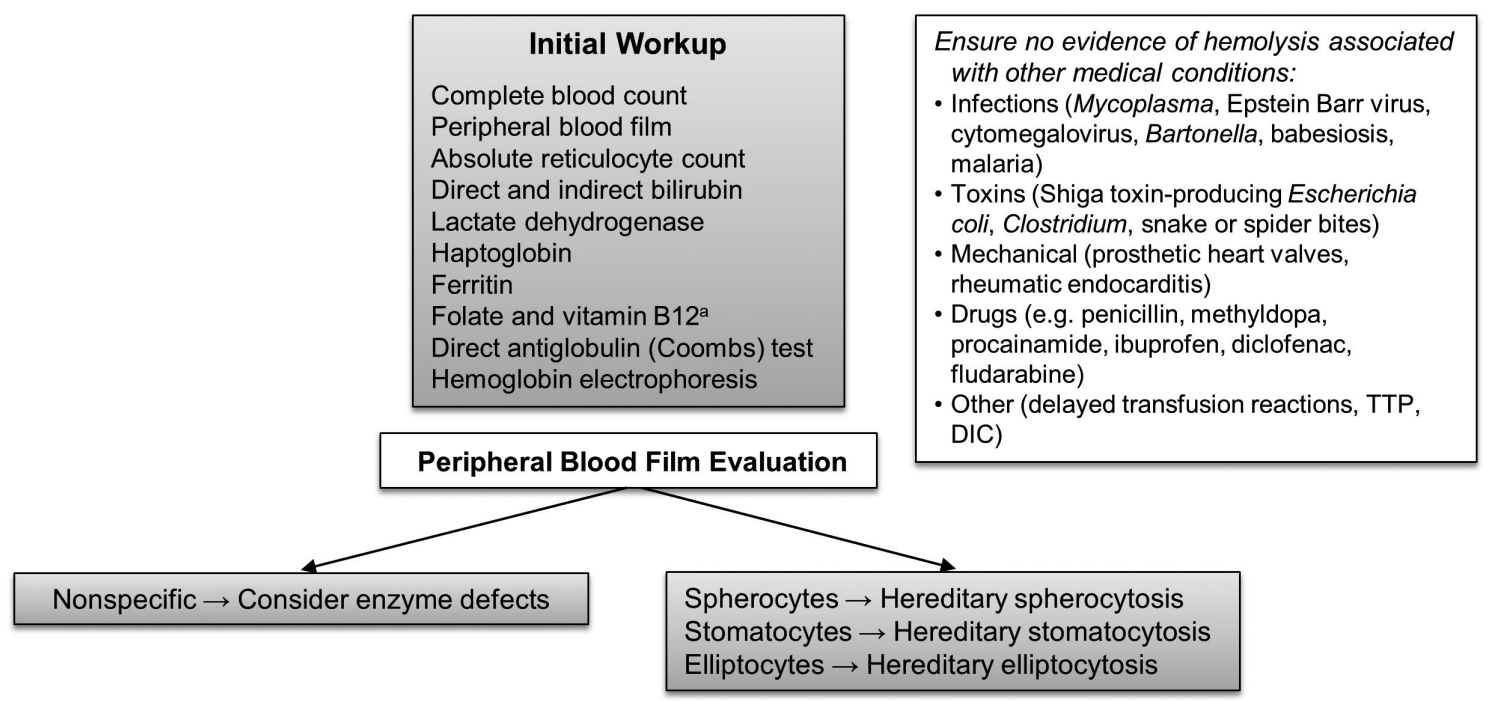

\section{Second-Pass Workup}

Glucose-6-phosphate dehydrogenase activity High-performance liquid chromatography ${ }^{\mathrm{b}}$ Flow cytometry for CD55 and CD59 for PNH

\section{Third-Pass Workup}

Pyruvate kinase enzyme activity PKLR molecular testing Osmotic fragility testing Eosin-5'-maleimide (EMA) binding Osmotic gradient ektacytometry Membrane protein analysis

\section{If Diagnosis Still Unknown, Consider:}

- Enzymatic activity and molecular testing for glucose phosphate isomerase (GPI), phosphofructokinase (PFK), triose phosphate isomerase (TPI), phosphoglycerate kinase (PGK), hexokinase (HK), and pyrimidine $5^{\prime}$-nucleotidase ( $\mathrm{P} 5 \mathrm{~N}$ ) deficiencies

- KLF1 molecular testing

- More sensitive DAT methods for DAT-negative AIHA

- Molecular testing and bone marrow examination and for congenital dyserythropoietic anemias (CDAs)

- Molecular testing for unstable hemoglobin variants

- Family studies and next generation sequencing (NGS) panels for complex cases

- Copper and ceruloplasmin measurement (poisoning, Wilson disease)

- ADAMTS13 measurement (congenital Upshaw-Schulman syndrome)

Figure 3. Our consensus, stepwise approach to laboratory workup of a patient with chronic hemolytic anemia. The initial workup includes hemolysis testing performed routinely. The second-pass workup is intended to rule out relatively common inherited entities (including hemoglobinopathies not identified in the initia workup) as well as paroxysmal nocturnal hemoglobinuria, particularly relevant if the patient presents in adulthood. The third-pass workup allows for identification of pyruvate kinase deficiency and red cell membrane abnormalities not diagnosed in prior steps. If this three-step workup is unrevealing, additional testing is recommended to diagnose particularly rare inherited and acquired causes of hemolytic anemia. The diagnostician may narrow or broaden the workup at each step as appropriate and as testing is available; for example, molecular PKLR and KLF1 testing can be reasonably performed earlier in the workup. Additionally, the clinician should be aware that many specialized tests are poorly standardized between laboratories. ${ }^{a}$ Deficiency may result in a hemolytic picture due to ineffective erythropoiesis; folate may be low in chronic hereditary anemias due to rapid cell turnover. 'Allows identification of most hemoglobinopathies. TTP: thrombotic thrombotic thrombocytopenic purpura; DIC: disseminated intravascular coagulopathy; PNH: paroxysmal nocturnal hemoglobinuria; DAT: direct antiglobulin test; ADAMTS13: a disintegrin and metalloproteinase with thrombospondin motifs 13. 
sent for additional genetic analyses including non-coding intronic region sequencing and copy number variation analyses.

\section{Impact of disease manifestations on treatment decisions}

Splenectomy and hematopoietic stem cell transplantation in pyruvate kinase deficiency

CASE: A 6-year old boy with genetically confirmed PKD presents to the hematology clinic for a discussion regarding his management, particularly for consideration of splenectomy. This is prompted by the complications of his disease thus far. In his lifetime he has received a total of ten red blood cell transfusions during episodes of symptomatic anemia in the setting of increased hemolysis with viral infections.

Supportive care for $\mathrm{PKD}$ is focused on transfusions and/or splenectomy. Approximately $60 \%$ of PKD patients are splenectomized, with a median hemoglobin rise of 1.6 $\mathrm{g} / \mathrm{dL} .{ }^{11}$ Splenectomy is often performed in patients with significant anemia, transfusion burden, and/or massive splenomegaly. The potential benefits and risks of splenec- tomy must be weighed against those of continued anemia and/or red blood cell transfusions. The risks of splenectomy, including post-splenectomy infections and thrombotic complications, have been extensively reviewed elsewhere. $^{40-43}$ The rate of venous thrombosis in PKD is $10 \%$, which is similar to the rate in other hemolytic disorders, while the reported rate of post-splenectomy sepsis may be as high as $7 \%$, which is higher than that reported in other cohorts of splenectomized individuals. ${ }^{36}$ The risk of sepsis is highest in the first year after surgery and in young children but is life-long. The decision to proceed with splenectomy should be delayed until after the age of 5 years, but this timing and the risk of post-splenectomy sepsis must be weighed against the risks of iron loading in patients treated with regular transfusions. Early referral to hematology and surgery for evaluation is advised to assess the pros and cons of splenectomy.

The majority of patients with PKD will experience an improvement in hemolysis with both an increased hemoglobin and decreased transfusion burden after splenectomy; however, patients have continued compensated hemolysis with an ongoing risk of severe anemia due to increased hemolysis with infections, aplastic crises associated with parvovirus, and bilirubin-related gallbladder dis-

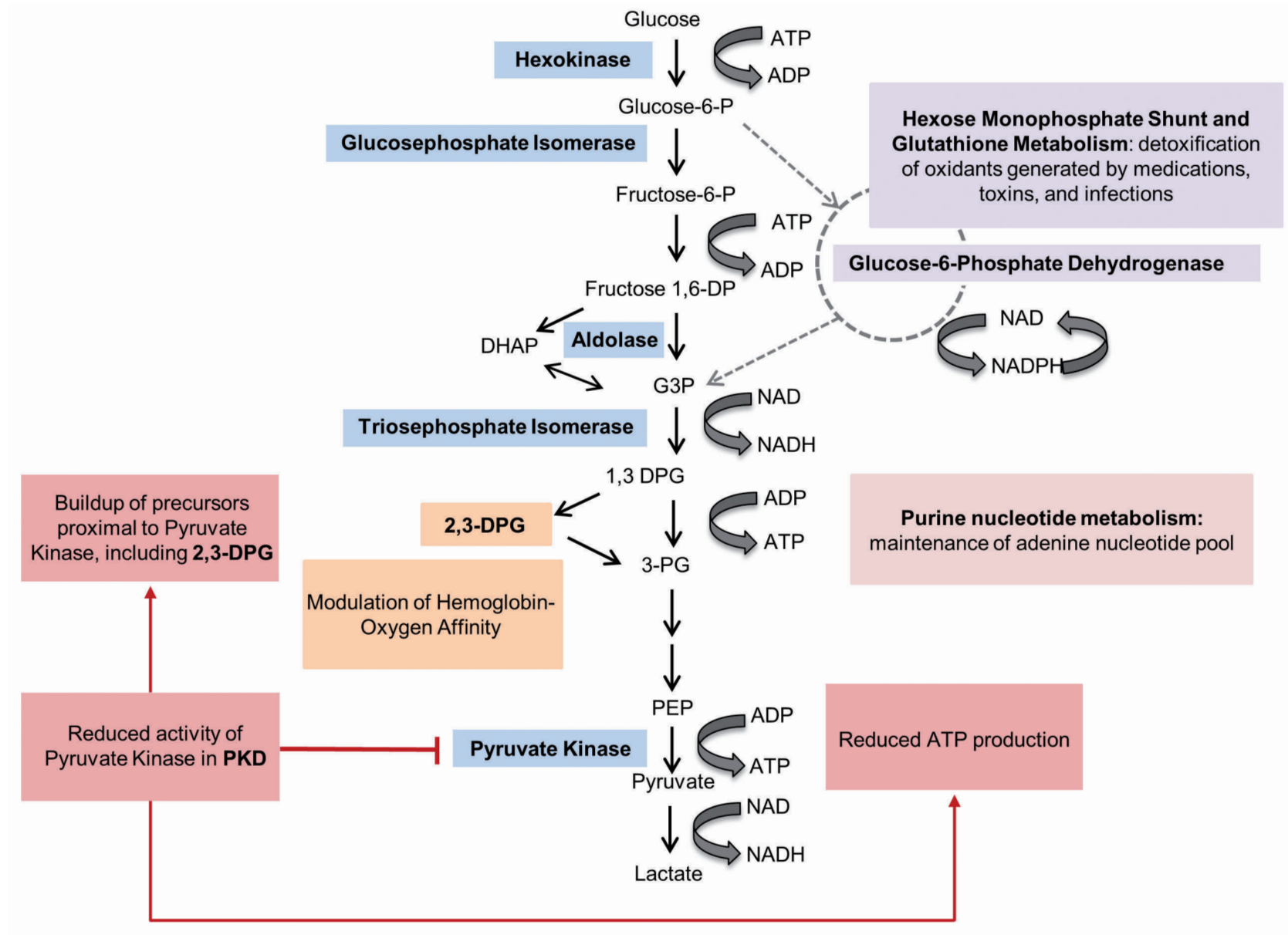

Figure 4. The glycolytic pathway. Deficiency of pyruvate kinase results in diminished ATP production as well as buildup of pathway intermediates proximal to pyruvate kinase, most notably 2,3-diphosphoglycerate. Modified with permission from Grace and Glader. ${ }^{57}$ Glucose-6-P: glucose-6-phosphate; Fructose-6-P: fructose-6-phosphate; Fructose 1,6-DP: fructose 1,6-diphosphate; DHAP, dihydroxyacetone phosphate; G3P: glucose-3-phosphate; 1,3-DPG: 1,3-diphosphoglycerate; 2,3-DPG, 2,3diphosphoglycerate; 3-PG: 3-phosphoglycerate; PEP: phosphoenolpyruvate. Blue: enzymes in glycolytic pathway that correlate with the more common glycolytic enzymopathies. 
ease. ${ }^{9,36,44}$ Approximately $15 \%$ of patients with PKD will be regularly transfused despite splenectomy. ${ }^{36}$ Although response to splenectomy is difficult to predict, those patients who continue to require transfusions despite splenectomy tend to have two drastic (e.g. non-missense) PKLR mutations and a higher pre-splenectomy rate of hemolysis. ${ }^{36}$

The decision to proceed with splenectomy is more common in US centers, whereas in European centers, more patients with PKD remain on transfusions and iron chelation with intact spleens. This practice variation likely reflects provider and patient preferences and values assigned to the potential risks and benefits of splenectomy. International expert guidelines recommend splenectomy in patients with $\mathrm{PKD}$ who receive regular transfusions or do not tolerate their anemia. Cholecystectomy may be recommended at the time of splenectomy given the persistent risk of bilirubin gallstones, but patients should be counseled about the risk of intrahepatic cholestasis and other complications. ${ }^{40}$ Full splenectomy is recommended given the reported poor hemoglobin response to partial splenectomy. ${ }^{45}$ Pre- and post-splenectomy vaccinations are critical for preventing sepsis, and antibiotic prophylaxis should be considered according to country-specific guidelines but is often considered lifelong. Post-splenectomy thromboprophylaxis can be considered in the immediate postoperative period in patients with additional thrombotic risk factors. If thromboprophylaxis is given, low-dose aspirin can be considered in adults with additional risk factors (including advanced age, a history of thrombosis, or cigarette smoking) until the platelet count is $<500 \times 10^{\circ} / \mathrm{L}$ and in children until the platelet count is $<1,000 \times 10^{9} / \mathrm{L}$.

Patients with a mildly low hemoglobin without symptoms of anemia, even with concurrent infections, can clearly avoid splenectomy. However, the management of many patients, like the one in this case, is not straightforward. He has received a considerable number of transfusions, possibly each time he has had a viral infection, and he likely has a high hemolytic rate and low hemoglobin at baseline. He would benefit from a post-splenectomy rise in hemoglobin, which could help him to avoid transfusions with illnesses going forward. Inquiring about agespecific signs and symptoms of fatigue will help to determine whether a chronically low hemoglobin is associated with daily symptoms, an additional reason to support moving forward with full splenectomy and cholecystectomy in this patient. Further complicating this discussion is the potential for future targeted treatments for $\mathrm{PKD}$, including mitapivat (AG-348), an oral small molecule allosteric activator of $\mathrm{PK}$ currently in phase III clinical trials in adults. If the patient has at least one missense PKLR mutation, consideration could be given to delaying splenectomy while awaiting potential regulatory approval of this treatment. Off-target mild aromatase inhibition may, however, delay the future availability of this treatment until adulthood. ${ }^{46}$

Curative treatment with stem cell transplantation has been pursued in a small number of patients with only 16 transplanted patients reported worldwide. These patients had a high rate of grade IV graft-versus-host disease and only a $74 \%$ cumulative survival at 2 years; however, they had variable ages at transplant, were given different types of transplants, and were treated with a variety of conditioning regimens. ${ }^{16}$ Transplant could be considered for the patient in this case, particularly with an HLA-matched unaffected sibling. In the rare case that transplantation is under serious consideration, splenectomy and its associated infectious and thrombotic risks should be avoided. Given that patients with two drastic PKLR mutations and a higher hemolytic rate are less likely to benefit from splenectomy or mitapivat, consideration should be given to a stem cell transplant and the availability of matched donors prior to splenectomy. However, given the available data regarding the risks of transplant versus supportive care and the treatments in development, including gene therapy, an approach of ongoing supportive care with splenectomy and/or regular red cell transfusions is recommended rather than stem cell transplantation in the majority of patients, including the patient in this case.

\section{Transfusion in pyruvate kinase deficiency}

CASE: $A B$ (age 38) and BB (age 35) are brothers with PKD diagnosed by enzyme assay and confirmed by genetic testing. Both had splenectomies in childhood, and subsequently had baseline hemoglobin levels of $8-9 \mathrm{~g} / \mathrm{dL}$ with $20-35 \%$ reticulocytes. In early childhood both brothers had similar burdens of fatigue and activity limitation attributable to PKD. In adulthood, the brothers have had divergent paths owing to decisions regarding regular transfusion.

$A B$ is relatively sedentary and is transfused once or twice a year, only for worsening symptoms of anemia. He is unemployed. He is concerned about iron overload and refuses regular transfusion with iron chelation due to side effects of chelation therapy in the past. He, therefore, resists red blood cell transfusions until he is clinically more symptomatic.

$B B$, in contrast, is an active, working, married man with four children. In order to maintain this active lifestyle, he is regularly transfused to keep his hemoglobin concentration above 10-11 $g / d L$. To prevent iron overload with regular transfusions, he takes an oral iron chelation drug.

Patients with PKD frequently live with chronic fatigue, poor exercise tolerance, and suboptimal work productivity. ${ }^{47}$ These symptoms may improve if the hemoglobin concentration is raised consistently with regular transfusions.

The decision to transfuse a patient with PKD is based on the patient's tolerance of anemia and demands of their lifestyle rather than an arbitrary hemoglobin threshold. In the above cases, two brothers with the same baseline hemoglobin and symptom level, the decision to regularly transfuse one but not the other was based on the impact of their disease on their quality of life. For the regularlytransfused sibling, red cell transfusions allow him to work, support his family, and live an active lifestyle; by contrast, his brother prioritizes avoiding the side effects of iron chelation and opts for on-demand transfusions only.

\section{The asymptomatic patient with severe anemia}

CASE: An 8-year-old child with transfusion-dependent congenital anemia of unclear etiology is diagnosed with $P K D$ following enzyme assay and confirmatory genetic testing, which demonstrated the presence of two deleterious non-missense mutations, c.721G>T (p.Glu241X) and c.284-2A>C (abnormal splicing), predicted to result in a complete absence of the $P K-R$ isoform. During the course of his diagnostic workup, his 2,3diphosphoglycerate levels were measured at over three times the upper limit of the reference range. He underwent splenectomy with resolution of transfusion dependency and a post-splenectomy baseline hemoglobin of $7.0 \mathrm{~g} / \mathrm{dL}$. Despite continued severe 
anemia following splenectomy, the patient excelled at school and extracurricular activities. He has maintained a normal social life into adulthood, married, and had children. As a 40-year old man, he underwent quality of life assessments, including the Functional Assessment of Chronic Illness Therapy Fatigue subscale [FACIT-F, final score of 48 (score range 0-52)] and the Functional Assessment of Cancer Therapy [FACT-G, score of 96 (score range 0-104)], confirming an excellent quality of life.

Despite his lack of complaints or need for transfusion, he was offered routine PKD screening (as detailed in Table 1) and was found to have a ferritin of $670 \mathrm{ng} / \mathrm{mL}$. Subsequent liver magnetic resonance imaging demonstrated an average liver iron content of $8.9 \mathrm{mg} / \mathrm{g}$ dry weight (reference range, $0.17-1.8 \mathrm{mg} / \mathrm{g}$ dry weight). After a discussion of the risks of iron overload he was initiated on chelation therapy with deferasirox.

This case illustrates several important points about the spectrum of disease in $\mathrm{PKD}$, most notably the poor correlation between symptoms and severity of anemia. In the case described, the patient's lack of symptoms despite his low hemoglobin was evident both from his high level of functioning and the results of two well-validated quality of life instruments. Patients may be less symptomatic than with other types of anemia for a given hemoglobin level, or occasionally entirely asymptomatic despite severe anemia. ${ }^{48}$ This is in part because 2,3-diphosphogylcerate, an important regulator of the oxygen affinity of hemoglobin, is increased in $\mathrm{PKD}$, which may enhance oxygen delivery. ${ }^{17,49}$ This increase occurs due to the metabolic block in the glycolytic pathway resulting in upstream accumulation of glycolytic intermediates (Figure 4). The resulting P50 and other patient-related factors, such as older age and concurrent medical problems, play a role in symptom burden. This is important to recognize as unnecessary transfusion of patients who are clinically well can be harmful due to iron loading.

The 2,3-diphosphogylcerate level obtained during the workup of the patient's anemia prior to the diagnosis of PKD is biochemical evidence consistent with what is observed clinically regarding his excellent tolerance of anemia. Although correlations have been demonstrated between the presence of two non-missense mutations and both more severe disease and worse health-related quality of life, ${ }^{11}$ genotype may poorly predict phenotype in some patients with PKD. ${ }^{50}$ The clinical manifestations of the disease are caused by the complex interactions of the PKLR genetic background, concomitant functional polymorphisms of other enzymes, posttranslational or epigenetic modifications, ineffective erythropoiesis and differences in splenic function. ${ }^{50}$ Varying degrees of compensatory expression of the $\mathrm{PK}$ isozyme normally expressed in leukocytes, PK-M2, in PK-R-deficient erythrocytes appears to influence the clinical severity of PKD as well..$^{51}$

Similar cases describing relatively asymptomatic PKD patients with even lower hemoglobin levels have been published, ${ }^{52}$ and indeed a better-than-expected tolerance of anemia in many patients likely explains the not uncommon delay observed in time to diagnosis. ${ }^{53}$ Further studies are needed to characterize the relationship between a given patient's 2,3-diphosphogylcerate levels, P50 oxygen dissociation curve, clinical phenotype, genotype, and patient-reported quality of life in PKD and in other congenital hemolytic anemias.

Lastly, this case highlights the occult iron overload common in PKD. Iron overload regularly occurs even in nevertransfused patients over a lifetime of chronic hemolysis, necessitating regular evaluation of iron status in all $\mathrm{PKD}$ patients. ${ }^{15}$ In the completely asymptomatic patient who may only visit the clinic annually, assessment of iron status and institution of appropriate chelation therapy is easy to overlook, potentially leading to development of endorgan damage.

\section{Special situations}

\section{Pregnancy in pyruvate kinase deficiency}

CASE: A 24-year old woman (gravida 1, parity 0) with a history of iron-deficiency anemia due to menorrhagia (hemoglobin 9.5-11.0 g/dL) and a clinical diagnosis of Gilbert syndrome presents in the 12th week of pregnancy with anemia and profound fatigue. On evaluation, physical examination is remarkable only for mild pallor and jaundice and a palpable spleen tip. Laboratory testing demonstrates hemoglobin $8.3 \mathrm{~g} / d \mathrm{~L}$, mean corpuscular volume $99 \mathrm{fL}$, absolute reticulocyte count 225×109/L, haptoglobin $<20 \mathrm{mg} / \mathrm{dL}$ (reference range $30-200 \mathrm{mg} / \mathrm{dL}$ ), lactate dehydrogenase $267 \mathrm{U} / \mathrm{L}$ (reference range 135-214 U/L), negative direct antiglobulin testing, and unremarkable red cell morphology on a peripheral blood film. Pyruvate kinase enzyme activity was reduced (3.9 U/g Hb [11-15.6]) and genetic testing revealed the presence of two missense mutations: $c .1456 \mathrm{C}>\mathrm{T}$ (p.Arg486Trp) and c.994G>A (p.Gly332Ser). Other congenital and acquired hemolytic conditions were ruled out by normal osmotic fragility, eosin-5-maleimide binding, ektacytometry, erythrocyte membrane protein content, hemoglobin electrophoresis, and CD55/59 antigen expression. During pregnancy the patient was closely followed by hematology and obstetrics staff. Because of severe fatigue, she was given a total of 15 units of packed erythrocytes during pregnancy (on average, a transfusion every 2 weeks) and four additional units at the time of delivery because of postpartum bleeding complications. Following delivery of a preterm but healthy infant, the mother's anemia improved to a hemoglobin of $9.5 \mathrm{~g} / \mathrm{dL}$.

Pregnancy is among the known triggers of hemolysis in PKD, along with infections and erythropoietic stressors. In addition to increased hemolysis, hydremia of pregnancy may further exacerbate anemia in pregnant women. Little is known about fertility and pregnancy in $\mathrm{PKD}$, except for a tendency towards an increased frequency of preterm births and miscarriages, and an increased transfusion need. $^{54,55}$ Folate supplementation is mandatory and we advise a minimum of $1 \mathrm{mg}$ daily during pregnancy. Fetal growth should be carefully monitored. 2,3-diphosphogylcerate levels result in a rightward shift of the oxygen dissociation curve of hemoglobin and symptoms may not correlate well with severity of anemia. As in non-pregnant patients, the decision to transfuse is guided by the patient's symptoms, rather than hemoglobin levels, and by fetal growth as measured by ultrasound.

Given that splenectomy partially ameliorates the anemia in PKD, anticipation of pregnancy in a young woman with PKD may be considered as a factor in favor of splenectomy prior to pregnancy. ${ }^{40}$

\section{Stigmatizing Icterus in pyruvate kinase deficiency}

CASE: A 14-year old boy with PKD who has been splenectomized arrives at the clinic for routine follow-up. He is able to be physically active to his satisfaction despite his anemia (baseline hemoglobin $9.0 \mathrm{~g} / \mathrm{dL}$ ). His primary complaint during this visit, as was the case in his past several visits, is severe jaundice of the 
skin and scleral icterus. He has been the victim of considerable bullying by other children in his new school over the past year due to the yellow tinge of his skin and eyes. His mother believes it is making him depressed and his grades have fallen over the same period. His total bilirubin is $3.9 \mathrm{mg} / \mathrm{dL}$. He is initiated on phenobarbital $15 \mathrm{mg}$ daily, resulting in a reduction of his bilirubin to $2.0 \mathrm{mg} / \mathrm{dL}$ and significant improvement in the icterus. At his next visit, the patient remarks that the bullying has stopped.

Patients with congenital hemolytic anemia often live with chronic icterus. This is not uncommonly a significant psychological burden and social stigma for patients with PKD with a considerable impact on quality of life. Schoolaged children and adolescents may face bullying and other negative social consequences. It is important for clinicians to recognize this and address the impact on their patients' quality of life. Depending upon the degree of impact on a patient's well-being, treatment may be indicated to address icterus. Low-dose, off-label phenobarbital (15-30 mg daily) could be considered as this agent induces hepatic bilirubin metabolism, although the potential side effects of this medication should be carefully explained to the patient and family. ${ }^{56}$ In especially severe cases, a regular exchange transfusion regimen can be considered to reduce bilirubin levels and alleviate icterus, although this approach was not uniformly agreed upon by all members of the working group.

\section{Disease burden over the lifespan}

CASE: A 54-year old woman with PKD presents for routine follow-up. She was diagnosed in childhood and was transfusiondependent until she underwent splenectomy at the age of 8. This alleviated her need for routine transfusions and she settled at a hemoglobin of 8.0-9.0 g/dL. Between the age of 8 and 49, she required red cell transfusions only five times: in the post-operative setting following cholecystectomy, during pregnancy, and during three acute infectious episodes that resulted in dramatic drops in her hemoglobin and hospitalization. During her late 40s, she noticed slowly worsening exercise tolerance despite no change in her baseline hemoglobin and at the age of 49 began receiving intermittent transfusions to treat fatigue. By the age of 52, she required regular transfusion of two units of red cells every 8 weeks to raise her hemoglobin above $10.0 \mathrm{~g} / \mathrm{dL}$. Over time, her tolerance of anemia has diminished further, resulting in a gradual reduction of the interval between transfusions. Currently she requires two units of red cells every 6 weeks. She does not smoke and has no underlying cardiopulmonary disease, including no evidence of pulmonary hypertension.

Patients with PKD are susceptible to hemolytic crises, typically occurring in the setting of acute viral or bacterial infection. ${ }^{41}$ Hemolytic crises may also be precipitated by pregnancy, surgery, or other major physiological stressors. During a hemolytic crisis, the hemoglobin concentration drops precipitously and transfusion is frequently required. Similarly, patients can develop aplastic crises secondary to parvovirus B19, which should be suspected in the setting of a hemoglobin drop with a significant reduction in the normally robust reticulocytosis. This possibility can be evaluated with parvovirus B19 serology (including IgM testing) or polymerase chain reaction testing.

The described scenario of a patient who is liberated from transfusions following splenectomy in childhood but decides to start regular transfusions once again in later adulthood due to anemia-related symptoms demonstrates a common but poorly-studied phenomenon in PKD: a reduced tolerance to the same degree of anemia as patients age. This phenomenon was universally recognized by working group members but has not been formally described in the literature. In this patient, an evaluation for cardiopulmonary disease, such as the pulmonary hypertension that can complicate PKD, is warranted. ${ }^{11,25}$ A decline in cardiopulmonary function also frequently occurs due to non-PKD-related disease or from age-related factors, which can reduce the patient's ability to compensate for the degree of anemia. Cases such as this one emphasize that transfusion independence in PKD may come and go throughout the patient's lifespan.

\section{Conclusions}

While the management of PKD may change in the near future with the promise of therapeutic advances on the horizon, recognition and diagnosis of the disease by hematologists and institution of proper monitoring and supportive treatments will remain important. This requires an understanding of the manifestations of disease as they relate to diagnosis, treatment, and impact on the patient's quality of life. With few distinctive clinical signs to suggest the disease, both a high index of suspicion and understanding of the limitations of diagnostic testing are needed to properly diagnose patients. Recognition that transfusions are symptom-directed and that iron overload and other complications are common regardless of the severity of the anemia is critical for proper care of $\mathrm{PKD}$ patients. The spectrum of disease in PKD is broad with symptoms varying between patients and within patients over time with age, supporting an individualized approach to monitoring and treatment.

\section{Acknowledgments}

HA is the recipient of the National Hemophilia FoundationShire Clinical Fellowship Award and the Harvard KL2/Catalyst Medical Research Investigator Training Award and the American Society of Hematology Scholar Award.

\section{References}

1. Beutler E, Gelbart T. Estimating the prevalence of pyruvate kinase deficiency from the gene frequency in the general white population. Blood. 2000;95(11):3585-3588.

2. Carey PJ, Chandler J, Hendrick A, et al. Prevalence of pyruvate kinase deficiency in northern European population in the north of England. Northern Region Haematologists Group. Blood. 2000;96(12):4005-4006.
3. Machado P, Manco L, Gomes C, et al Pyruvate kinase deficiency in sub-Saharan Africa: identification of a highly frequent missense mutation (G829A;Glu277Lys) and association with malaria. PLoS One. 2012;7(10):e47071.

4. Ayi K, Min-Oo G, Serghides L, et al. Pyruvate kinase deficiency and malaria. N Engl J Med. 2008;358(17):1805-1810.

5. Selwyn JG, Dacie JV. Autohemolysis and other changes resulting from the incubation in vitro of red cells from patients with congenital hemolytic anemia. Blood. 1954;9(5): 414-438.

6. Valentine WN, Tanaka KR, Miwa S. A specific erythrocyte glycolytic enzyme defect (pyruvate kinase) in three subjects with congenital non-spherocytic hemolytic anemia. Trans Assoc Am Physicians. 1961;74:100110.

7. Necheles TF, Finkel HE, Sheehan RG, Allen DM. Red cell pyruvate kinase deficiency. 
The effect of splenectomy. Arch Intern Med. 1966;118(1):75-78

8. Mentzer WC Jr., Baehner RL, SchmidtSchonbein H, Robinson SH, Nathan DG. Selective reticulocyte destruction in erythrocyte pyruvate kinase deficiency. J Clin Invest. 1971;50(3):688-699

9. Nathan DG, Oski FA, Miller DR, Gardner FH. Life-span and organ sequestration of the red cells in pyruvate kinase deficiency. $\mathrm{N}$ Engl J Med. 1968;278(2):73-81.

10. Matsumoto $\mathrm{N}$, Ishihara $\mathrm{T}$, Nakashima $\mathrm{K}$, Miwa S, Uchino F. Sequestration and destruction of reticulocyte in the spleen in pyruvate kinase deficiency hereditary nonspherocytic hemolytic anemia. Nihon Ketsueki Gakkai Zasshi. 1972;35(4):525-537.

11. Grace RF, Bianchi P, van Beers EJ, et al. Clinical spectrum of pyruvate kinase deficiency: data from the Pyruvate Kinase Deficiency Natural History Study. Blood. 2018;131(20):2183-2192.

12. Ferreira P, Morais L, Costa R, et al. Hydrops fetalis associated with erythrocyte pyruvate kinase deficiency. Eur J Pediatr. 2000;159 (7):481-482.

13. Hennekam RC, Beemer FA, Cats BP, Jansen G, Staal GE. Hydrops fetalis associated with red cell pyruvate kinase deficiency. Genet Couns. 1990;1(1):75-79.

14. van Beers E, Kuo K, Morton D, et al. Health related quality of life and fatigue in patients with pyruvate kinase deficiency. Blood. 2018;132(Suppl 1):4807.

15. van Beers EJ, van Straaten S, Morton DH, et al. Prevalence and management of iron overload in pyruvate kinase deficiency: report from the Pyruvate Kinase Deficiency Natural History Study. Haematologica. 2019;104(2): e51-e53.

16. van Straaten S, Bierings M, Bianchi P, et al. Worldwide study of hematopoietic allogeneic stem cell transplantation in pyruvate kinase deficiency. Haematologica. 2018;103 (2):e82-e86.

17. Grace RF, Zanella A, Neufeld EJ, et al. Erythrocyte pyruvate kinase deficiency: 2015 status report. Am J Hematol. 2015;90(9):825-830.

18. Yang H, Merica E, Chen Y, et al. Phase 1 single- and multiple-ascending-dose randomized studies of the safety, pharmacokinetics, and pharmacodynamics of AG-348, a firstin-class allosteric activator of pyruvate kinase $\mathrm{R}$, in healthy volunteers. Clin Pharmacol Drug Dev. 2019;8(2):246-259.

19. Grace RF, Rose C, Layton DM, et al. Safety and efficacy of mitapivat in pyruvate kinase deficiency. N Engl J Med. 2019;381(10):933944.

20. Garcia-Gomez M, Calabria A, Garcia-Bravo $M$, et al. Safe and efficient gene therapy for pyruvate kinase deficiency. Mol Ther. 2016;24(7):1187-1198.

21. Thompson AA, Walters MC, Kwiatkowski J, et al. Gene therapy in patients with transfusion-dependent beta-thalassemia. $\mathrm{N}$ Engl J Med. 2018;378(16):1479-1493.

22. Yamaguti-Hayakawa GG, Ozelo MC. Gene therapy: paving new roads in the treatment of hemophilia. Semin Thromb Hemost. 2019;45(7):743-750.

23. Bianchi P, Fermo E, Glader B, et al. Addressing the diagnostic gaps in pyruvate kinase deficiency: consensus recommendations on the diagnosis of pyruvate kinase deficiency. Am J Hematol. 2019;94(1):149161

24. Hipkins R, Thompson J, Naidoo P, Bishay E, Djearaman M, Pratt G. Images in haematol- ogy. Paravertebral extramedullary haemopoiesis associated with pyruvate kinase deficiency. Br J Haematol. 2009;147 (3):275

25. Bachmeyer C, Khalil A, Kerrou K, Girot R, Gounant V. Idiopathic pulmonary arterial hypertension in a patient with pyruvate kinase deficiency and paravertebral extramedullary hematopoiesis. Ann Hematol. 2009:88(6):603-605.

26. Plensa E, Tapia G, Junca J, Perez R, Castella E, Marti S. Paravertebral extramedullary hematopoiesis due to pyruvate kinase deficiency. Haematologica. 2005;90(Suppl): ECR32

27. Goh DH, Lee SH, Cho DC, Park SH, Hwang $\mathrm{JH}$, Sung JK. Chronic idiopathic myelofibrosis presenting as cauda equina compression due to extramedullary hematopoiesis: a case report. J Korean Med Sci. 2007;22(б):10901093.

28. Gouliamos A, Dardoufas C, Papailiou I, Kalovidouris A, Vlahos L, Papavasiliou C. Low back pain due to extramedullary hemopoiesis. Neuroradiology. 1991;33(3):284 285.

29. Sawhney H, Weedon J, Gillette P, Solomon W, Braverman A. Predilection of hemolytic anemia-associated leg ulcers for the medial malleolus. Vasa. 2002;31(3):191-193.

30. Aessopos A, Kati M, Tsironi M, Polonifi E Farmakis D. Exchange blood transfusion for the treatment of leg ulcerations in thaassemia intermedia. Haematologica. 2006; 91(5 Suppl):ECR11.

31. Alavi A, Kirsner RS. Hemoglobinopathies and leg ulcers. Int J Low Extrem Wounds. 2015;14(3):213-216

32. Jansen G, Koenderman L, Rijksen G, Punt K, Dekker AW, Staal GE. Age dependent behaviour of red cell glycolytic enzymes in haematological disorders. Br J Haematol. 1985;61 (1):51-59.

33. Warang P, Kedar P, Ghosh K, Colah R Molecular and clinical heterogeneity in pyruvate kinase deficiency in India. Blood Cells Mol Dis. 2013;51(3):133-137.

34. Pissard S, Max-Audit I, Skopinski L, et al Pyruvate kinase deficiency in France: a $3-$ year study reveals 27 new mutations. Br J Haematol. 2006;133(6):683-689.

35. Manu-Pereira Mdel M, Gonzalez-Roca E, van Solinge WW, et al. Pyruvate kinase deficiency and severe congenital hemolytic anemia in a double heterozygous patient with paternal transmission of an early germ-line de novo mutation. Am J Hematol. 2015;90 (12):E217-219.

36. Grace RF, Bianchi P, van Beers EJ, et al. The clinical spectrum of pyruvate kinase deficiency: data from the Pyruvate Kinase Deficiency Natural History Study. Blood. 2018;131(20): 2183-2192

37. Bagla S, Bhambhani K, Gadgeel M, Buck S, Jin JP, Ravindranath Y. Compound heterozy gosity in PKLR gene for a previously unrecognized intronic polymorphism and a rare missense mutation as a novel cause of severe pyruvate kinase deficiency. Haematologica. 2019:104(9):e428-e431.

38. Lezon-Geyda K, Rose MJ, McNaull MA, et al. Pklr intron splicing-associated mutations and alternate diagnoses are common in pyruvate kinase deficient patients with single or no Pklr coding mutations. Blood. 2018;132(Suppl 1):3607.

39. van Oirschot BA, Francois JJ, van Solinge WW, et al. Novel type of red blood cell pyruvate kinase hyperactivity predicts a remote regulatory locus involved in PKLR gene expression Am J Hematol. 2014:89(4):380384

40. Iolascon A, Andolfo I, Barcellini W, et al. Recommendations regarding splenectomy in hereditary hemolytic anemias. Haematologica. 2017;102(8):1304-1313.

41. Grace RF, Mark Layton D, Barcellini W. How we manage patients with pyruvate kinase deficiency. $\mathrm{Br} J$ Haematol. 2019:184(5):721-734.

42. Kristinsson SY, Gridley G, Hoover RN, Check D, Landgren O. Long-term risks after splenectomy among 8,149 cancer-free American veterans: a cohort study with up to 27 years follow-up. Haematologica. 2014:99(2):392-398.

43. Rodeghiero F, Ruggeri M. Short- and longterm risks of splenectomy for benign haematological disorders: should we revisit the indications? $\mathrm{Br} J$ Haematol. 2012;158(1):16-29.

44. Zanella A, Fermo E, Bianchi P, Valentini G. Red cell pyruvate kinase deficiency: molecular and clinical aspects. Br J Haematol. 2005;130(1):11-25.

45. Sandoval C, Stringel G, Weisberger J, Jayabose S. Failure of partial splenectomy to ameliorate the anemia of pyruvate kinase deficiency. J Pediatr Surg. 1997;32(4):641-642

46. Kung C, Hixon J, Kosinski PA, et al. AG-348 enhances pyruvate kinase activity in red blood cells from patients with pyruvate kinase deficiency. Blood. 2017;130(11):13471356.

47. Grace RF, Cohen J, Egan S, et al. The burden of disease in pyruvate kinase deficiency: patients' perception of the impact on healthrelated quality of life. Eur J Haematol. 2018;101(6):758-765.

48. Oski FA. Clinical consequences of enzyme deficiencies in the erythrocyte. Ann Clin Lab Sci. 1971;1(2):177-183.

49. Lakomek M, Winkler $\mathrm{H}$, Pekrun A, et al. Erythrocyte pyruvate kinase deficiency. The influence of physiologically important metabolites on the function of normal and defective enzymes. Enzyme Protein. 1994;48(3):149-163.

50. Zanella A, Fermo E, Bianchi P. Chiarelli LR, Valentini G. Pyruvate kinase deficiency: the genotype-phenotype association. Blood Rev. 2007:21(4):217-231.

51. Diez A, Gilsanz F, Martinez J, Perez Benavente S, Meza NW, Bautista JM. Lifethreatening nonspherocytic hemolytic anemia in a patient with a null mutation in the PKLR gene and no compensatory PKM gene expression. Blood. 2005;106(5):1851-1856.

52. Aksu T, Yarali N, Fermo E, et al. A case with pyruvate kinase deficiency remarkably sensitive to heat. I Pediatr Hematol Oncol. 2018;40(7):e458-e460

53. Aydin Koker S, Oymak Y, Bianchi P, et al. A new variant of PKLR gene associated with mild hemolysis may be responsible for the misdiagnosis in pyruvate kinase deficiency. J Pediatr Hematol Oncol. 2019:41(1):e1-e2.

54. Amankwah KS, Dick BW, Dodge S. Hemolytic anemia and pyruvate kinase deficiency in pregnancy. Obstet Gynecol. 1980;55(3 Suppl):42S-44S.

55. Wax JR, Pinette MG, Cartin A, Blackstone J. Pyruvate kinase deficiency complicating pregnancy. Obstet Gynecol. 2007;109(2 Pt2):553-555.

56. Capron JP, Erlinger S. Barbituates and biliary function. Digestion. 1975;12(1):43-56.

57. Grace RF, Glader B. Red blood cell enzyme disorders. Pediatr Clin North Am. 2018;65 (3):579-595 